\title{
EAl Endorsed Transactions

\section{Prognostic Modelling for Smart cities using Smart Agents and IoT: A Proposed Solution for Sustainable Development}

\author{
Shafqat Ul Ahsaan ${ }^{1}$, Ashish Kumar Mourya ${ }^{2, *}$ \\ ${ }^{1}$ Department of Computer Science and Engineering, Jamia Hamdard, Delhi-110062 \\ ${ }^{2}$ Department of Computer Science and Engineering, Gautam Buddha University, India
}

\section{Abstract}

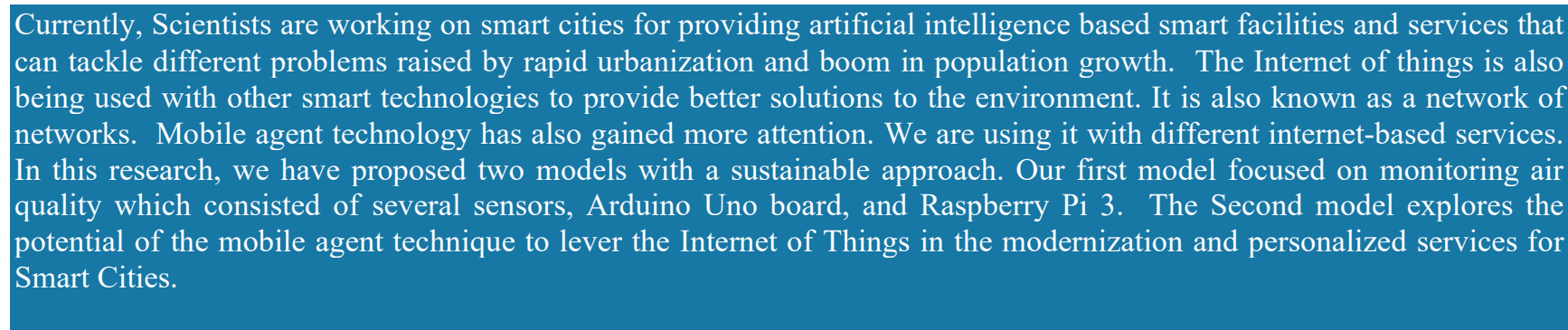

Keywords: Smart City, Internet of Things (IoT), Software Agent, Mobile Agent, Artificial Intelligence (AI), Digital Initiative, Arduino Uno board, Raspberry Pi 3

Received on 21 February 2021, accepted on 13 May 2021, published on 13 May 2021

Copyright (C) 2021 Shafqat Ul Ahsaan et al., licensed to EAI. This is an open access article distributed under the terms of the Creative Commons Attribution license, which permits unlimited use, distribution and reproduction in any medium so long as the original work is properly cited.

doi: 10.4108/eai.13-5-2021.169916

*Corresponding author. Email: Ashishkumarmourya@gmail.com

\section{Introduction}

The idea of the high-tech smart city is moderately new, and it will soon take over to knowledge city, digitalized city, and feasible city. Although, it has been used regularly exclusively after 2012, although the conversation about its architecture and thought in recent years. There is a lack of unanimity about it. Smart city term has covered the different sub-themes such as smart urbanization, smart financial system, sustainability, and smart environment, high-tech technology, energy conservation, smart mobility, smart Medicare, and so on [1]. With the advancement on the internet of things devices and their application, mobile spectrum, broadband communication, next-generation, cloud-based technology, etc.; informatization has a propensity of higher smarter phase. Recently some corporate houses issued the concept of "Smart globe". Smart globe believes that sensing devices are embedded in public places like public transport station, bridges, Subways, roads, buildings, rainwater harvesting systems, dams, mercantile equipment, and healthcare devices, and then physical facilities can be professed, so Digital technology extends into the physical world, creating an "Internet of Things". Moreover, the Internet of Things may relate to networking to numeral the social community and physical system [2]. The human being, the machine, electronic devices can be managed in the incorporated system through networking and cloud computing, so the human's invention and life can be managed more accurately and energetically to get smarter, hoist resource usage and productivity, and improve the association between human and environment. The smart (high-tech) city also relies on the IoTs, sensing devices, and cloud computing to provide an extensive network of linked electronic devices. Smart sensing devices and large-scale data analytics to enable the move from the internet of things to real-time control.

Smart devices-based machinery has revolutionized the digital era and became an important field of life particularly 
in the context of sustainability. Internet of Things (IoT) is a collection of objects that are connected in such a way that they form a network. These networks of objects can communicate with each other utilizing the internet, mobile phones, sensors that have been implanted at specific areas, Radio Frequency Identification (RFID), General Phone Radio Service (GPRS), and computers [1-2]. These objects communicate with the help of unique addresses that allow them to be verified and addressed over the network. The objects in the network are assigned with a specific task and send reports to the users [3]. Internet of things technology is applying in smart cities as a key component. For instance (see Figure 1), to offer user-friendly services, the data capture by digital home appliances including refrigerators, washing machines are share and use in a smart home environment [2].

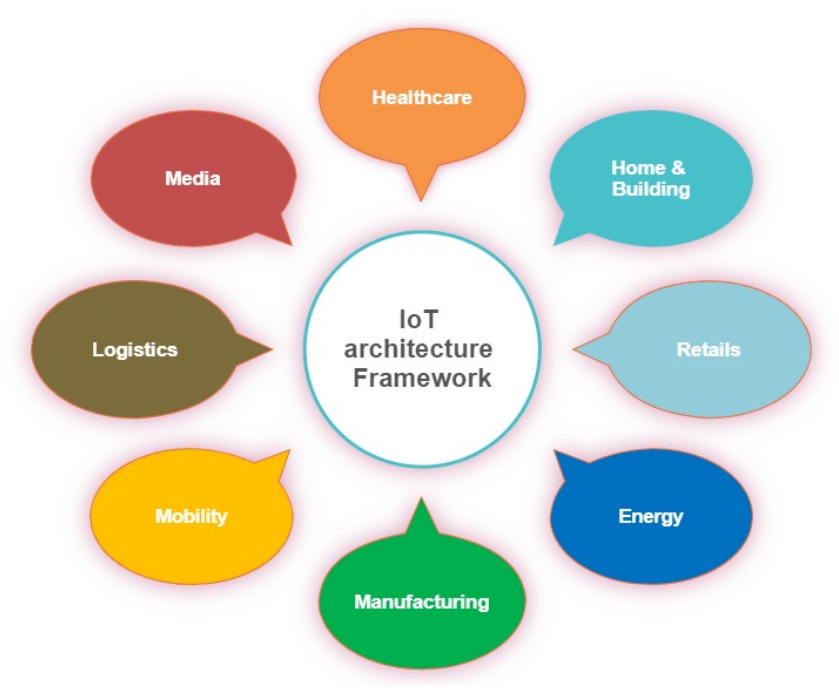

Figure 1. IoT application Domain

Smart devices-based machinery has revolutionized the digital era and became an important field of life particularly in the context of sustainability. Internet of Things (IoT) is a collection of objects that relate to each other in such a way that they form a network. These networks of objects can communicate with each other by means of internet, mobile phones, sensors that have implanted at specific areas, Radio Frequency Identification (RFID), General Phone Radio Service (GPRS) and computers [1-2]. The advancement in technology with the passage of time has changed the overall scenario. These objects communicate with the help of unique addresses that allow them to be verified and addressed over the network. The objects in the network are assigned with a specific task and send reports to the users [3].

Mobile agents (software agent technology) have some advantages that have boosted the Internet of Things technology. The key role of Mobile Agents is to minimize networking capacity, encapsulate protocols to resolve network latency. They can perform autonomous tasks during accumulation, which would otherwise require large configuration. However, the drawback of this technology is the use of unique technology and host environment is expected by many agent systems that make it difficult to use them and entail that especial skills are required in the formation of agents. Mobile Agents are executable (runtime) entities which can switch from one host to another along with the software applications' internal state. This means that an executing agent on the current system will interrupt its execution and continue with another system. A special case of moving code integrating remote evaluation with internal state preservation is mobile agents $[4,5]$.

\section{Challenges and need for loT in Smart City}

As smart city concepts continue flourishing tirelessly, their challenges need to be considered from beginning for population growth, economic development, and social progress to follow the same path. Every smart city scheme includes high-tech lighting, imaginative transport systems, and high-tech utility metering for electricity and water. All these emerging technologies and integrations fully depend on sensor-centred data gathering and analysis. While the various benefits of smart city initiatives remain, due to specific city requirements and different interpretations of implementation principles, several difficulties when it comes to deployment. Some important challenges that need to be tackled when we talked about the smart city concept are listed below:

- High-Speed Internet Connectivity

- Low-cost IoT devices

- Data security

- Governance

- GPS enabled Transport services

- Interoperability

Implementation of sensing devices in smart cities like IoT is an extraordinarily complex practice. It needs a lot of strategies and sometimes a large number of investments. This is because the Internet of Things system consists of many diverse components. Implementation of each of the IoT components bears different challenges along with it, so we must be careful during applying these smart city management policies. Therefore, the implementation of IoT in the smart city is one of the biggest challenges because it needs time and huge money. That is why policymakers across the world are trying to make the atmosphere more users friendly to develop and working on the cheapest devices. This Manuscript has worked on the following objectives:

I. The proposed study provides a solution for smart city development with sustainable approaches. 
II. What are the main challenges and hurdles in between the development of smart cities?

III. The Proposed study also compared different studies that have been carried out so far.

IV. The Proposed study also built two models for smart cities.

V. Analysis of State of the Art about Smart City technology

\section{Comparative Study of Different Models for Smart City}

In literature, there are more than enough definitions for smart cities, but the universal definition has not been framed yet. It looks that Digital and Smart City both the terms are frequently used in educational research to delineate the "intelligence" of a city. The outline regarding the term; how a city is said to be smart is covered in different research publications. What are the characteristics of a smart city? There are almost all researchers from every field who mainly focus on sustainable development of cities with elevated reliance on a permanent progressive route map for the development of cities. A setup to consider the model of smart cities is built on eight significant features: people and communities, leadership and organization, advanced machinery, policy context, control, economy, infrastructure, and natural surroundings [1].

These factors act as the foundation of a consolidative framework that puts forward the guidelines and outline to neighbouring governments for envisaging smart city projects. The model and connection of the smart city with the digital city is based on the core content of implementation procedures, the tackle to buildability, and the impact of its progress. The smart city fundament is the primary step to set up the smart city skeleton and structural design on the whole; its progressive configuration and the area wise precision of tracing the properties as a foundation of the development of smart city planning united with the entire amenities and organization allied to the smart city agenda [2].

In [3], the authors' put forward an approach that can be used for the computation of smart city indices. Although, they choose heterogeneous indicators, include a vast amount of information. The paper is concerned with the calculation of allocated weights and the approach used here is based on fuzzy logic for the considered indicators. The authors'[4] presented the paper with a new concept of the u-eco-city into a check and concentrate on whether the u-eco-city is an astounding smart and sustainable city that represents an ultimate 21 st-century city model or just a branding deception. Eco-city proposal is the striving step of the $21 \mathrm{st}$ century. Eco-cities are considered as zero-carbon, carbonneutral, low-carbon, ubiquitous-eco, and sustainable, highlight their position as per sustainability. This study focuses on ubiquitous-eco-city (u-eco-city). Here in [5], the authors' discussed in detail the favourable circumstances using ICT and considered it as a promising technology to lessen the use of energy in our cities. The authors' developed an analytical model in which the study of ICT opportunities is pooled with a typology of domestic functions, i.e. all the daily activities that require energy. The energy that is required for domestic functions is evaluated with the help of a consumption-based point of view. This paper [6] proposes a theoretical agenda to study and examine two principal cases from the US and Asia. The main objective of this paper is to focus on the progress in building an efficient smart city by amalgamating a variety of practical viewpoints with contemplation of smart city characteristics. Here a model is developed to carry out case studies probing how smart cities were being put into practice in San Francisco and Seoul Metropolitan City. The study's experimental outcome implies that efficient, sustainable smart cities appear under methods that are influential in which communal and economic sectors are brought together to coordinate their activities and resources on an open advanced policy.

In [7], the authors' have developed a conceptual model that makes it simple to interpret how neighbouring administrations build up demand-side guidelines tools that inspire the growth and dispersal of sustainable-driven transformations that boost limited fiscal growth. The authors in [8] give a broad understanding of the idea of a smart city while elaborating the categorization of relevant application domains, like buildings, living, government, natural assets and liveliness, transportation and mobility, economy, and people. It also investigated the dispersal of smart proposals employing an experimental study meant for exploring the percentage of province enclosed by a city's paramount performance to the full potential domains of smart projects and accepting the function that various demographic, geographical, trade and industry and municipal variables may be affecting the arrangement move to build a smarter city. In this study, results disclose that the advancement patterns of a smart city are extremely dependent on its confined circumstances. In [9], current studied opus and currently programs for the sustainability and the conditions suitable for living in cities and monitored indicators that are required for effective monitoring. In this study, the development through indices was proposed through principal component analysis, and the real time data is used as an alternative of historical data as the essential information that one may build a set of indicators to elucidate the proposal. This article [10] summarizes and gives a detailed introduction regarding eco-town based urban-development implantation in the city North-western Europe. The authors' emphasized the development of ecotowns and the rules and regulations, processes, and models with the help of which the eco-towns were started. 
In [11], the main focus is to find the role of innovative, intelligent urban machinery and tools in the developmental progress of smart cities. The authors put forward a comprehensive review regarding the applications of the smart city framework while coming across the emerging practices of ubiquitous eco-cities. This paper [12] highlights the differences in public transport among the two famous cities of the United Kingdom like Newcastle and southern Brazil city Florianopolis. The study made a comparison of models, measures for the progressive growth of Florianopolis as a sustainability model in South America. Hence, a detailed investigation of changes, models that were built and the similarities and differences have been verified to discover the behaviours that direct social and political process in the field of urban sustainability. This paper gives a proportional depiction of social and financially viable indicators like the gross domestic product (GDP) per capita, price increases, employment, as well as the historical population evolution of the two cities.

Within [13], the authors' performed a relative case-study of three Asian cities like Penghu in Taiwan, Seoul in South Korea, and Tianjin in China and revealed the special effects of multiple nationwide methods to eco-city progress. In their study, they have compared the Asian cities with two European cities, Freiburg (Germany) and Samso (Denmark). The investigation makes out four transforming antecedents of the growth of an eco-city in Asia, which are (1) the presence of a dedicated local public authority, (2) deployment of a countrywide approach and policy, (3) a combination of national potential, and (4) commerce activity the continuous engagement of local citizens. In [14], '16' sets of city measurement framework (8 smart-city and 8 municipal sustainability evaluation models) composed of '958' indicators in total by separating the indicators into three groups and 12 divisions. The paper put forward a comparison of modern technologies and smartness that must be implemented to make a city smart to urban sustainability frameworks. A common motive of smart cities is to improve sustainability with help of technologies. In this [15] paper, an indicator agenda for the estimation of low carbon city (LCC) was known from the viewpoint of Energy pattern, societal and livelihood, Economic, inner-city mobility, Solid waste, Carbon and Environment, and Water. A complete assessment scheme was engaged for LCC ranking utilizing the entropy weighting factor method. The standard standards for LCC documentation were also known. The model was practiced in 10 international cities to grade them according to their low-carbon levels. A comparative study was done at multiple points of trade and industry, societal, and green development improved the entire study. The outcome proved that Stockholm, Vancouver, and Sydney position at the top level than the standard value, signifying these cities gained a grade value far above the ground in the emission of low-carbon growth.

\section{IoT based Model for Monitoring Air Quality}

The basic outline of the first model is drafted in Figure 2. It is composed of several sensors, an Arduino Uno board, and Raspberry Pi 3. Raspberry Pi 3 is a convenient and alarming Single Board Computer (SBC) that includes an ARM processor and works on Linux Operating System. It is an adaptable system-on-chip (SoC) panel that can be used for multiple purposes. In Raspberry Pi 3, BCM2837 chip is used which is based on 64-bit ARM v7 Quad-Core Processor and runs across operating systems like Windows 10, Ubuntu Mate, and Raspbian. The BCM2837 chip operates at a clock rate of $1.2 \mathrm{GHz}$ and is employed using $1 \mathrm{~GB}$ of RAM and 40 unmitigated GPIO pins [16,17]. It provides internet access through BCM43143 Wi-Fi Module. Raspberry Pi is the core component of the architecture that controls the system. The sensors are being installed at multiple locations within the vicinity for detecting the concentration of multiple gases like carbon dioxide, oxygen, nitrogen dioxide, ozone gas, etc. These gas sensors are linked to an Arduino Uno board which is connected to Raspberry pi 3 via Universal Serial Bus (USB) cable. The data captured by the detector/sensors is directly fed to Raspberry Pi 3 in communication with the Arduino Uno board to the cloud. The MQ9, MQ7, MQ8, MG811, and MQ135 gas sensors are analog sensors that measure the gas concentration in the region where they are being installed [18]. 


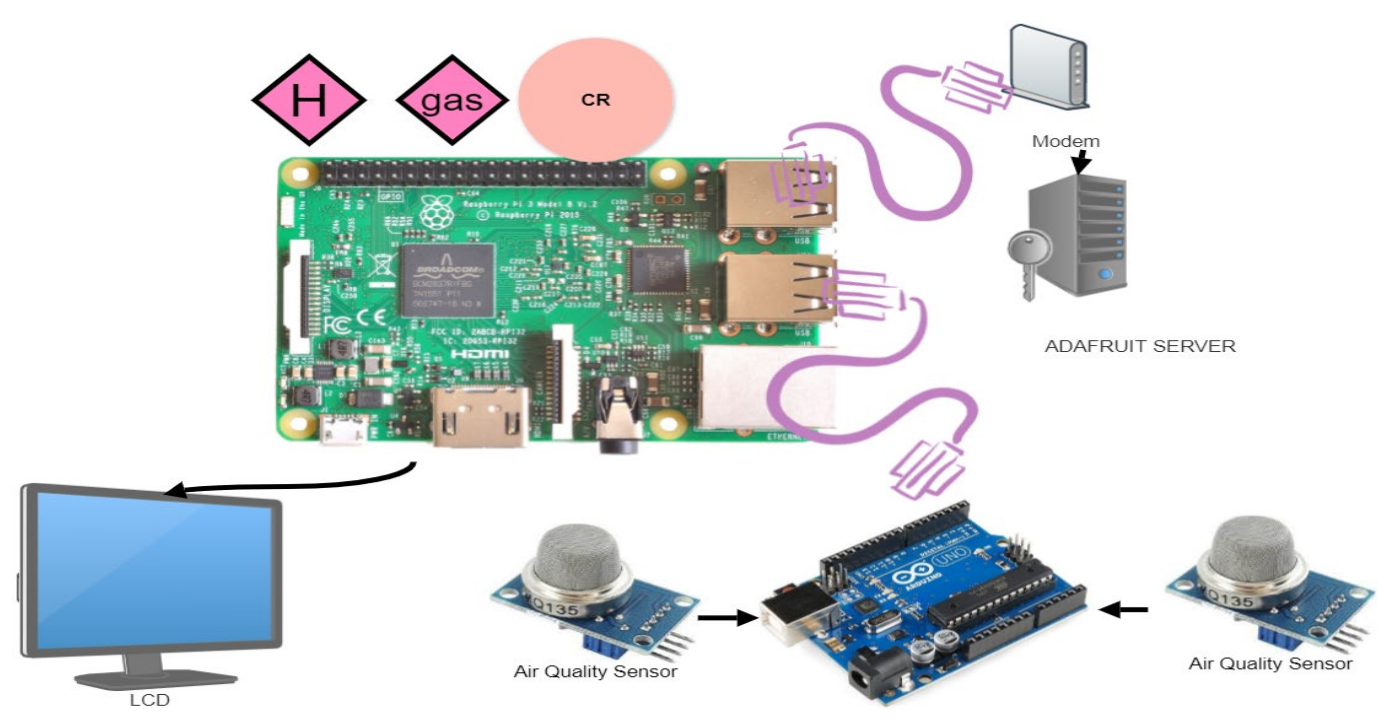

Figure 2. Air quality monitoring System

\subsection{Arduino Uno Board}

Arduino Uno is an inexpensive microcontroller chipboard based on ATMEGA-32P that can be configured simply with Raspberry Pi and provides a very efficient Analog to Digital Conversion (ADC). Arduino Uno board has the feature like a USB interface, 14 digital I/0, 6 analog inputs primarily for reading analog sensors but can also be used as GPIO pins, which allow interfacing with various other boards [19]. Each of the digital pins is capable to perform input or output operations, using functions like pin Mode to digital read and digital Write. Arduino Uno microcontroller board can be programmed using a simple integrated development environment using $\mathrm{C}$ or $\mathrm{C}++$. Raspberry Pi 3, model is enabled with Wi-Fi having double the performance power as compared to its existing generations. The proposed architecture makes use of lightweight Message Queuing Telemetry Transport (MQTT) protocol. The MQTT protocol smoothens the way of communication among sensors and clients. The data captured by the sensors are sent to the cloud through the internet and is accessible to clients. The data is visualized over the console by making use of device id [20].

\subsection{Raspberry Pi 3}

Raspberry $\mathrm{Pi} 3$ is a convenient and Single Board Computer (SBC) that includes an ARM processor and works on Linux Operating System. It is an adaptable system-on-chip (SoC), multifunctioning board. In Raspberry pi 3, BCM2837 chip is used which is based on 64-bit ARM v7 Quad-Core Processor and operates on software systems like Windows 10, Ubuntu Mate, and
Raspbian. The chip operates at a clock rate of $1.2 \mathrm{GHz}$ and is employed with $1 \mathrm{~GB}$ of RAM and 40 unmitigated GPIO pins. It provides internet access utilizing the BCM43143 Wi-Fi unit [18]. It is fixed with 4 USB ports,3.5 $\mathrm{mm}$ audio jack, 1 full High-Definition Multimedia Interface (HDMI) port, 1 Ethernet port, the display interface, and compound video camera interface. It provides a Micro SD slot which allows the users to store software like operating systems and some necessary drivers. Raspberry Pi 3 has a permanent ESP8266 Wi-Fi module that is appropriate for connecting Wi-Fi to the model through the UART serial connection. It is loaded with some exciting features like $802.11 \mathrm{~b} / \mathrm{g} / \mathrm{n}$ protocol, Wi-Fi Direct, and a consolidated TCP/IP protocol stack $[19,20]$.

\subsection{Sensing Unit}

The sensing unit contains an adequate number of gas sensors implanted at different locations. There are different types of gas detecting sensors such as MQ7, MQ8, MQ9, MG811, and MQ135. These sensors are extremely sensitive to carbon dioxide, ozone, nitrogen dioxide, etc. MQ135 is widely used for the finding of alcohol, $\mathrm{CO} 2, \mathrm{NH} 3$, and smoke with the minimal response time.

\subsection{Software Architecture}

It mainly consists of Node-Red and Integrated Development Environment. 
Node-Red is a convenient, open-source, flow-based development tool for visual programming developed by IBM. It is widely used for IoT based applications. It is a commonly used tool that allows IoT developers to merge APIs, hardware devices, and online services with ease. Node-Red is loaded with inbuilt libraries consisting of thousands of nodes and flows that permit the user to hook up all types of services and devices. A simple click mechanism is offered by the Node-Red to set up the flows by IoT developers to a lightweight execution environment [21].

\subsubsection{Integrated Development Environment}

Arduino based programs can be compiled in any software processing language that is provided with a compiler for the translation of programming code into the machine understanding form. Integrated Development Environment (IDE) can run on any hardware or software platform forms the foundation for Arduino [20]. It proves to be extremely influential for the people who are professionals in the field of project development, programmers, and researchers to build up different kinds of Arduino based projects by making use of multiple types of sensors. Arduino IDE is software for which the source code is freely available, licensed to permit modifications and redistribution of its source code, created from the incorporated development environment for data manipulation [21]. As we know that IDE is platform-independent, hence it can be executed on Linux and Windows-based operating systems. IDE has some attractive features like a toolbar for common functions, a massage area, and a text console. The programming languages supported by Arduino IDE are $\mathrm{C}, \mathrm{C}++$ which can be used to write programs within IDE.

\section{Proposed Smart city architecture with IoT by using Mobile Agent}

The Second model that we have introduced based on mobile agent technology for enabling interoperability and world-wide intellect with intelligent stuff in the Web of Things as shown in figure 3 , with assorted small, batteryoperated devices that consumes very less power where the systems spread across different systems that relate to each other from end-to-end and obligations. We consider the necessities to permit mobility operators in the IoT against various contexts: the intelligent stuff, the mobility agent, and the arrangement. Smart objects like IoT devices are loaded with digital elements, such as detectors, transducers, and actuators in each place of the smart city. All of this electronic machinery should be identifiable and accessed as resources of the digital stuff. These actuators in the vicinity where they are installed route the captured information makes them capable to comprehend their circumstances and to enhance the way of decision making on the fly and hence promote better and intelligent decisions. Smart stuff in the Internet of Things backing multiple interface models, like centralized communication, publish and subscribe messaging, eventdriven communication, and simulcast messages.

Digitalized stuff can participate in every condition and various networking techniques such as intranet work and internet-based communications across a disparate set of devices connected. Smart peripherals in IoT need to carry a lot of distributed training models: macro programming languages, code relocation, task offloading, cyber foraging, and virtual machines. Shared resources digital objects keep their support and potential, which comprise captured and refined data, object's competence, and besides host-based resource agents. These sources can be utilized in cooperation with additional stuff and operators in their procedures. The mobile agents preserve their status, uncovered using smart objects. As the condition of the task is loosely coupled into a hardware piece of equipment, the status of the task is cacheable, and agents offer durability in connection with failures".

The resource segment that is implanted in the vicinity states where the agent drifts across the system with the meticulous relocation guidelines given in the metadata section. We can make use of any relocation policy, for example, 1) the agent calls on every object scheduled in the neighboring source section only one time 2) the agent believes the neighboring source segment as a loop flowing among the devices, 3) the agent communicates with every object simultaneously 4) the neighboring source segment registers gateways or proxies, which allocates the agent to many distant smart objects. The relocation strategy can also be measured as a system resource. On every occasion the neighboring source part is mentioned, the smart objects depend on the information framework, like the network configuration warehouse for relocation directions. This mobile agent relocation process needs, at the lowest, that the agent is replicated in the host-based equipment and launch to the updated host, where the status is first reorganized by performing the compute-intensive task. Then the host enrolls the agent into the system server or directory catalyst. At this point, the improved agent status is open to the elements of the system and further objects gain the right to use the updated status in the host that is not existing before. Following the registration, an acceptance is sent to the earliest, which permits the host to delete the agent against its memory and set the previously used resources free. 

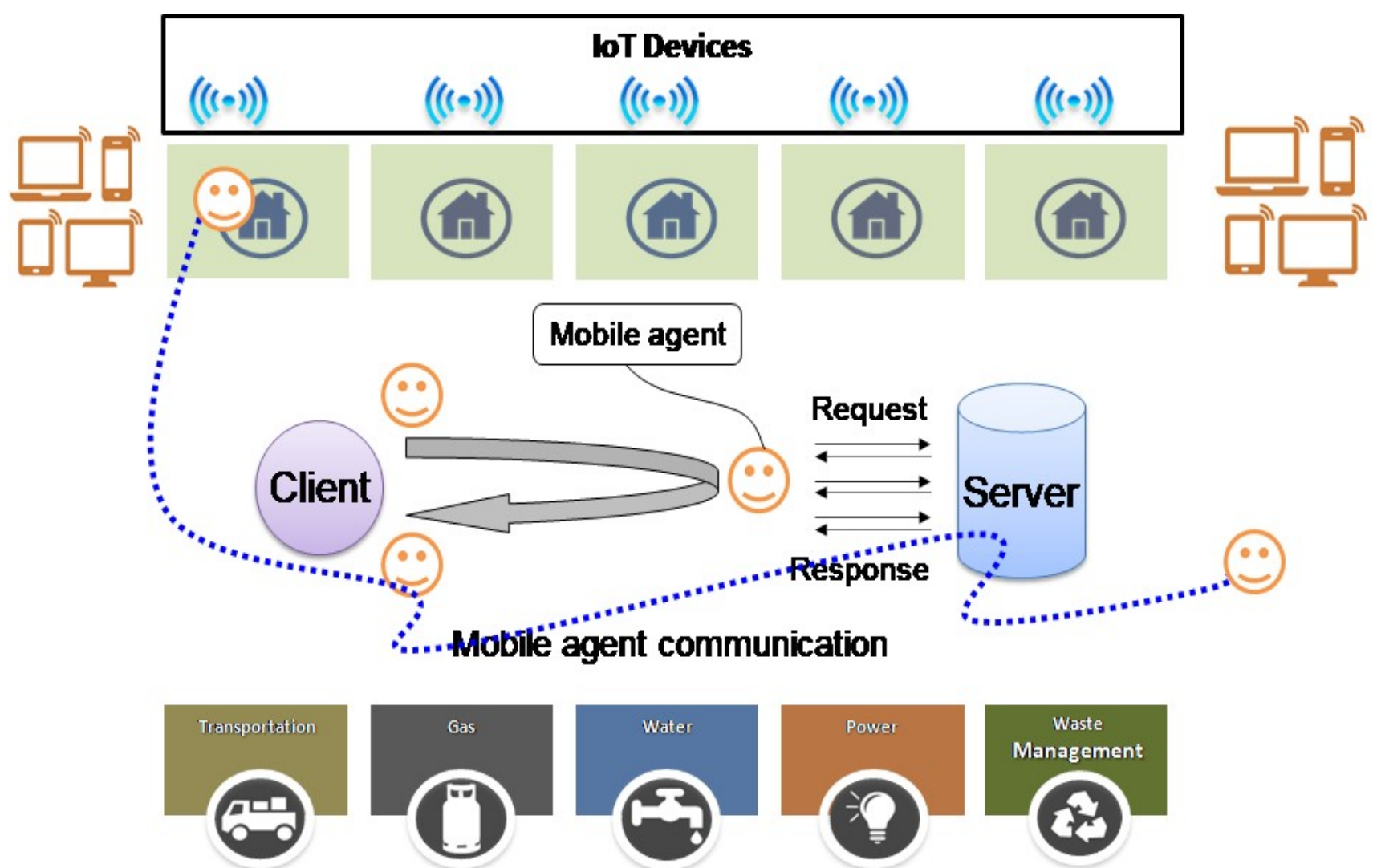

Figure 3. Mobile Agents based proposed model with loT

\section{Conclusion}

Smart city projects have been proven their importance for a high-tech living society. IoT devices with a high-speed internet connection have been used in a smart city. In this work, we proposed two architectures one of them uses mobile agent-based technology to configure the Internet of Things in a smart city. The mobile agent technology is executed on a platform that is wholly distributed and fault-tolerant to make the whole concept stronger. The characteristics of decentralization and load distribution among different kinds of multi-source devices could interact with each other on a peer-to-peer system to provide the services to clients. These functionalities may include day-to-day tasks as controlling electronic appliances remotely and cleverly or navigating around a remote location. The Proposed architectures also include execution of services using speech commands and speech to text prompts. Our proposed architectures also find few major applications in a smart high-tech city situation where unknown people such as guests may use services without taking help from others. The proposed models may show to be scalable \&robust and can accommodate to a big city.

\section{References}

[1] Conference: Bolívar, M. P. R. (2016, June). Mapping dimensions of governance in smart cities: Practitioners versus prior research. In Proceedings of the 17th International Digital Government Research Conference on Digital Government Research (pp. 312324).

[2] Journal: Bunce, S. (2016). Pursuing urban commons: Politics and alliances in community land trust activism in East London. Antipode, 48(1), 134-150.

[3] Journal: Castelnovo, W., Misuraca, G., \&Savoldelli, A. (2016). Smart cities governance: The need for a holistic approach to assessing urban participatory policymaking. Social Science Computer Review, 34(6), 724-739.

[4] Conference: Cocchia, A. (2014). Smart and digital city: A systematic literature review. In Smart city (pp. 13-43). Springer, Cham.

[5] Conference: Marsh, J., Molinari, F., \& Rizzo, F. (2016). Human smart cities: a new vision for redesigning urban community and citizen's life. In Knowledge, information and creativity support systems: Recent trends, advances and solutions (pp. 269-278). Springer, Cham.

[6] Journal: Ismagilova E, Hughes L, Rana NP, Dwivedi YK. "Security, Privacy and Risks within Smart Cities: Literature Review and Development of a Smart City 
Interaction Framework" [published online ahead of print, 2020 Jul 21]. Inf Syst Front. 2020;1-22. doi:10.1007/s10796-020-10044-1

[7] Journal: Md. Humayun Kabir, An overview of the Internet of Things (IoT) and IoT Security, Research Gate, June 2020.

[8] Conference: Liesbet van Zoonen, Privacy concerns in smart cities, Government Information Quarterly 33 (2016) 472-480

[9] Journal: Ali M A Abuagoub, "IoT Security Evolution: Challenges and Countermeasures Review", International Journal of Communication Networks and Information Security (IJCNIS) Vol. 11, No. 3, December 2019

[10] Journal: Aditya Tiwary, Manish Mahato, Abhitesh Chidar, Mayank Kumar Chandrol, Mayank Shrivastava, Mohit Tripath, "Internet of Things (IoT): Research, Architectures and Applications", International Journal on Future Revolution in Computer Science \& Communication Engineering, ISSN: 2454-4248 Volume: 4 Issue: 3

[11] Conference: Se-Ra Oh, Young-Gab Kim, Security Requirements Analysis for the IoT, (2017)

[12] Book Chapter: Butt, Talal \&Afzaal, Muhammad, "Security and Privacy in Smart Cities: Issues and Current Solutions", (2019), 10.1007/978-3-03001659-3_37.

[13] Conference: IS Farahat, AS Tolba, Mohamed Elhoseny, Waleed Eladrosy, Security in Smart Cities: Models, Applications, and Challenges, Springer (2019), Pages 117-142

[14] Conference: Fadi Al -Turjman, HadiZahmatkesh, Ramiz Shehroze; "An overview of security and privacy in smart cities' IoT communications",First published: 08 July 2019

[15] Journal: Barun, Benjamin C. M. Fung, Farkhund Iqbal, Babar Shah, "Security and privacy challenges in smart citie" Sustainable Cities and Society 39 (2018): 499-507.

[16] Website: "Internet of Things". URL: www.wikipedia.com. Date of access:20/10/2020

[17] Conference: Kumar, S. and Jasuja, A., 2017, May. Air quality monitoring system based on IoT using Raspberry Pi. In 2017 International Conference on Computing, Communication and Automation (ICCCA) (pp. 1341-1346). IEEE.

[18] Conference: Kiruthika, R. and Umamakeswari, A., 2017, August. Low-cost pollution control and air quality monitoring system using Raspberry $\mathrm{Pi}$ for Internet of Things. In 2017 International Conference on Energy, Communication, Data Analytics and Soft Computing (ICECDS) (pp. 2319-2326). IEEE.

[19] Journal: Maksimović, M., Vujović, V., Davidović, N., Milošević, V. and Perišić, B., 2014. Raspberry Pi as Internet of things hardware: performances and constraints. design issues, 3(8).

[20] Journal: Vujović, V. and Maksimović, M., 2015. Raspberry $\mathrm{Pi}$ as a Sensor Web node for home automation. Computers \& Electrical Engineering, 44, pp.153-171

[21] Conference: Nayyar, A. and Puri, V., 2016, March. A review of Arduino board's, Lilypad's \& Arduino shields. In $20163 \mathrm{rd}$ International Conference on Computing for Sustainable Global Development (INDIACom) (pp. 1485-1492). IEEE 